\title{
基于苯炔苯及苂光探针萠自组装单分子膜的制备及苯胺检测
}

\author{
胡金婷 ${ }^{a}$ 刘暘 ${ }^{b}$ 杨凯 $^{a}$ 石建兵 ${ }^{b}$ 佟斌 ${ }^{b}$ \\ 支俊格*, $a$ 董宇平*, \\ $\left({ }^{a}\right.$ 北京理工大学化学学院 北京 100081$)$ \\ $\left({ }^{b}\right.$ 北京理工大学材料学院 北京 100081$)$
}

\begin{abstract}
摘要 利用重氮基的光分解活性、采用自组装的方法将带有功能基团 $\mathrm{NH}_{2}$ 的 4-(4-氨基苯乙炔基)苯共价键接在石英基 片上制备了共轭单分子膜; 利用膜上 $\mathrm{NH}_{2}$ 的反应活性，通过酰胺化反应或自组装的方式将稠环萠修饰在单分子膜上, 构筑了含有萠荧光探针的稳定发光超薄膜; 该薄膜能够与电子受体硝基苯胺间因相互作用形成电荷转移络合物而使其 苂光猝灭, 并且该薄膜对硝基苯胺同分异构体的荧光猝灭响应有明显的差异, 其中对硝基苯胺对功能薄膜的猝灭效应 最为明显，其次是邻硝基苯胺和间硝基苯胺; 且具有明显的浓度依赖性.

关键词 共轭单分子膜; 芘; 重氮基光化学反应; 发光性能; 硝基苯胺的检测
\end{abstract}

\section{Fabrication of Self-Assembled Monolayer Based on Phenylethynyl- benzene and Fluorescent Probe Pyrene and Detecting for Nitroanilines}

\author{
$\mathrm{Hu}$, Jinting $^{a} \quad$ Liu, Yang ${ }^{b} \quad{\text { Yang, } \mathrm{Kai}^{a} \quad \text { Shi, Jianbing }}^{b} \quad{\text { Tong, } \text { Bin }^{b}}^{b}$ \\ Zhi, Junge* ${ }^{* a}$ Dong, Yuping*,b \\ $\left({ }^{a}\right.$ College of Chemistry, Beijing Institute of Technology, Beijing 100081) \\ $\left({ }^{b}\right.$ College of Materials, Beijing Institute of Technology, Beijing 100081)
}

\begin{abstract}
Self-Assembled monolayers (SAMs), a new type of organic ultra-thin films, show a lot of interesting properties in chemistry, physics, optics and electronic transport, and have potential application in biomaterials, nanoelectro-mechanical systems, sensors, and so on. In this paper, a full-conjugated unit-containing SAM ended with a functional amino group $\left(\mathrm{NH}_{2}\right)$ was fabricated by self-assembly and using a light-sensitive diazonium-containing compound 4-(4-amino phenylene ethynyl) benzenic diazonium salt (APEB- $\mathrm{N}_{2}^{+}$). The APEB- $\mathrm{N}_{2}^{+}$was easily deposited on a pre-treated quartz substrate with negative charges by electrostatic interaction and then grafted on the surface of a quartz substrate through covalent bond after the decomposition of diazonium group under UV irradiation, and a stable SAM based on phenylethynylbenzene was obtained. Furthermore, based on the reactivity of the functional group $\mathrm{NH}_{2}$, the fluorescent probe pyrene is anchored on the surface of the full-conjugated SAM through two approaches: amidation reaction between amino group on the SAM and carboxyl group of 1-pyrenebutyric acid, and self-assembly of $\mathrm{N}_{2}^{+}$, which is transferred from $\mathrm{NH}_{2}$ on $\mathrm{SAM}$, and electronegative $\mathrm{COO}^{-}$of 1-pyrenebutyric acid in basic aqueous solution. The obtained ultra-thin films with fluorescence probe pyrene exhibit luminescent property because of the emission of excimer of pyrene chromophore, which is mainly due to the formation of the $\mathrm{g}$-g conformation of pyrene for the induction effect of the full-conjugated SAM. The electron-acceptor nitroanilines (NAs) can quench the fluorescence of the obtained ultra-thin film because of electron and energy transfer between the electron-rich pyrene and NAs. The fluorescence quenching varies obviously for the different isomers of NA, and the quenching efficiency decreases according to the sequence of $p$-NA, $o-\mathrm{NA}, m-\mathrm{NA}$, because the electronic effect varies obviously when the relative position of electron withdrawing group $\mathrm{NO}_{2}$ and donating group $\mathrm{NH}_{2}$ of $\mathrm{NA}$ are different. The fluorescence of the ultra-thin film decreases with the increasing of the NA concentration.
\end{abstract}

Keywords conjugated self-assembled monolayer; pyrene; photochemistry of diazonium salts; luminescent property; detection of nitroaniline

\section{1 引言}

自组装单分子膜(Self-assembled monolayers, SAMs) 是基于化学或物理相互作用在基底表面自发构成排列
规整的单分子层有机超薄膜 ${ }^{[1 \sim 3]}$, 具有成膜条件易控、 均匀一致、高密堆积、低缺陷等特性; 并可预先设计，通 过精确的化学控制, 获得特定功能表面 ${ }^{[4 ~ 12]}$. 稠环芳烃 萠因其典型的 $\pi$ 共轭体系而具有独特的发光性质，被广

* E-mail: zhijunge@bit.edu.cn; chdongyp@bit.edu.cn

Received May 25, 2012; published July 27, 2012.

Supporting information for this article is available free of charge via the Internet at http://sioc-journal.cn.

Project supported by the National Natural Science Foundation of China (Nos. 20504005, 21074012).

项目受国家自然科学基金(Nos. 20504005, 21074012)资助. 
泛用于苂光探针及发光材料的研究中 ${ }^{[13 \sim 18]}$, 其中用于 自组装单分子膜的制备得到关注. Jen 等研究发现在 $\mathrm{Au}(111)$ 基底上采用不同取代基的萠自组装都能够形成 规整的 SAMs, 且 SAM 的性质与温度及间隔基有关 ${ }^{[8]}$; Gibbs 等报道了含有氨基和叠氮基双功能团的 SAMs, 并探讨了对组装在 SAM 上的两种官能团比例的影响因 素, 为制备多功能化表面的 SAMs 提供了一种新思路 ${ }^{[10]}$; 还有理论计算金表面长链烷基硫醇自组装单分子膜表 面电势的变化规律的报道 ${ }^{[12]}$. 因此, SAMs 为研究表面 与界面现象提供了理想方法, 在材料防腐、化学生物传 感器方面有广泛的应用前景; 同时, SAMs 具有纳米级 结构, 可作为光电装置元件或制备阵列传感器 ${ }^{[17 ~ 26] . ~}$

Ricco 等将 $\mathrm{Cu}^{2+}$ 固定在端基为羧基的 SAMs 上, 构 成对有机磷能选择性、可逆检测的敏感膜 ${ }^{[19]}$; Fang 等设 计合成了一系列具有特异响应性的自组装单分子膜, 用 来检测金属离子、硝基化合物、有机溶剂及气氛等, 并 研究了薄膜的结构特性、被检测物的物理化学性质对其 检测灵敏性的影响 ${ }^{[17,18,20 ~ 22]}$; 近期, 他们以 $\alpha$-萠取代三 聚噻吩为荧光发色团制备了光化学稳定的发光单分子 膜, 该薄膜对硝基芳香化合物蒸汽的检测灵敏性与被检 物蒸汽压直接相关; 并且, 此膜与水溶液中的 2,4,6-三 硝基苯酚因吸附作用并形成电荷转移络合物而对其具 有选择性灵敏检测 ${ }^{[18]}$; 另外, 他们将共轭聚合物引入单 分子膜, 探讨了所得功能薄膜对有机溶剂气氛的响 应 ${ }^{[23]}$. Nesterov 等将四聚苯撑乙炔齐聚物通过柔性链连 接在基片上，并在其端基修饰上醛基取代葱，该单分子 超薄膜会因醛基反应前后所引起的电子微环境的微小 扰动而发光不同, 实现了对能与醛基进行化学反应的 $L-$ 半胱氨酸的灵敏检测 ${ }^{[24]}$. 还有基于嗍酸和糖之间相互 作用制备多糖阵列并用于识别糖和蛋白质相互作用的 研究 ${ }^{[25]}$ 以及微波催化制备单分子薄膜的报道等 ${ }^{[26]}$.

本文采用自组装的方法、利用重氮基团的光反应活 性, 将共轭的苯炔苯结构以共价键接的方式引入石英基 片上制备了共轭单分子膜, 利用单分子膜上 $\mathrm{NH}_{2}$ 的反应 活性, 进一步功能化引入苂光探针萠, 研究了不同修饰 方式构筑的功能超薄膜的发光性能, 并初步探讨了该薄 膜对硝基苯胺同分异构体的菼光检测响应.

\section{2 结果与讨论}

\section{1 共轭单分子膜的制备}

因重氮基团反应活性较高, 易分解, 所以先以三氮 烯保护起来, 制备了化合物 4-[4-(3,3-二乙基三氮烯)-苯 乙炔基]氨基苯(2)(Scheme 1); 在制备薄膜时将三氮烯 在氟嗍酸/乙腈的溶液中室温分解为 4-(4-氨基苯乙炔)苯 基重氮盐(APEB- $\mathrm{N}_{2}^{+}$). APEB- $\mathrm{N}_{2}^{+}$溶液在 $235,320,362$ $\mathrm{nm}$ 出现了三个吸收峰(Supporting Information, Figure $\mathrm{S} 1)$, 分别对应于苯环的 $\pi-\pi *$ 电子跃迁、与重氮基共轭的 苯炔苯结构以及与苯炔苯基团共轭的重氮基的特征吸
收峰 ${ }^{[27]}$.

在单分子膜制备过程中，将预处理的带有负电荷的 石英基片浸入 APEB- $\mathrm{N}_{2}^{+}$溶液中, 因正负电荷间静电相 互作用，带正电荷的重氮基吸附在负电的石英片表面， 紫外光辐照下光敏的重氮基发生分解, 产生的芳基阳离 子与石英片表面的羟基作用生成共价键，得到 4-(4-氨 基苯乙炔)苯单分子膜(Figure 1a, 1b) ${ }^{[27 ~ 29]}$.

对 APEB- $\mathrm{N}_{2}^{+}$在乙腈溶液及单分子膜状态下重氮基 分解动力学的研究发现: 两种情况下重氮基都有较快的 分解速度; 但在乙腈溶液比单分子膜中具有更大的光分 解速率常数及较小的分解半衰期 (分别为 $45 \mathrm{~s}$ 和 $168 \mathrm{~s}$ ); 这是因为单分子膜中 $\pi-\pi$ 相互作用使共轭的 4-(4-氨基苯 乙炔)苯基之间排列规整, 形成了有序超分子聚集体 ${ }^{[30]}$, 增强了稳定性. 单分子膜上重氮基完全分解需要 $7 \mathrm{~min}$; 所以单分子组装膜制备时选择紫外光辐照时间为 10 $\min$, 以确保重氮基完全分解而转化成共价键 ${ }^{[27 ~ 30]}$.
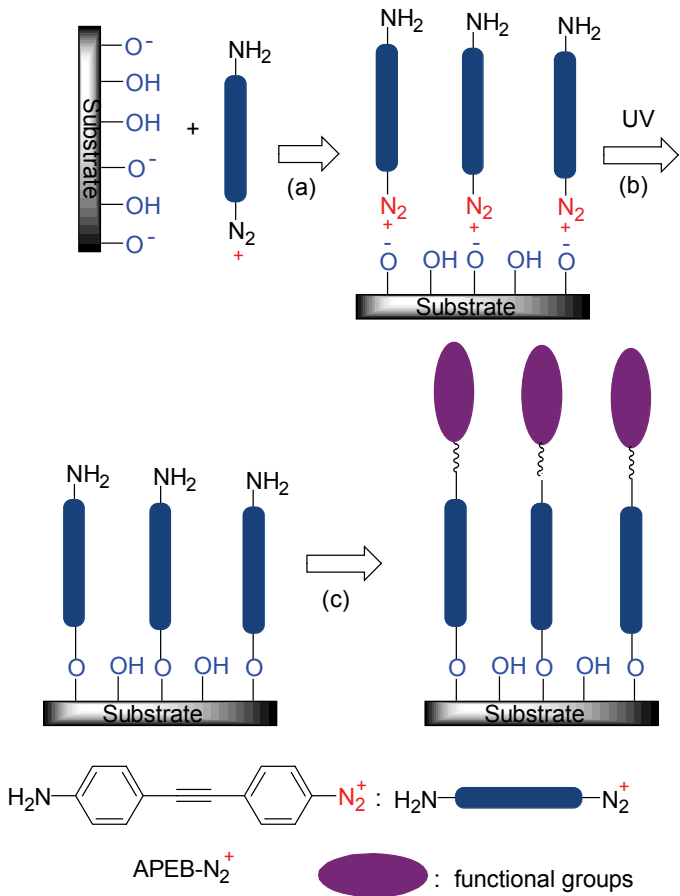

图 1 功能超薄膜的制备示意图

Figure 1 Fabrication strategy of functional ultra-thin monolayer

4-(4-氨基苯乙炔)苯单分子膜在 $235 \mathrm{~nm}$ 出现较弱的 紫外吸收, 在 $366 \mathrm{~nm}$ 出现了强而宽的紫外吸收(Figure 2 曲线 b), 归因于与苯炔苯共轭的重氮基团的电子跃迁; 紫外光辐照后, 重氮基分解, $366 \mathrm{~nm}$ 处重氮基吸收峰消 失, $300 \mathrm{~nm}$ 处出现了苯炔苯的紫外吸收(Figure 2 曲线 c), 因没有了重氮基的共轭作用, 该吸收峰与其溶液相比略 有蓝移; 同时, 光分解后的薄膜在 $400 \sim 700 \mathrm{~nm}$ 可见光 区也有明显的吸收, 主要源于自组装单分子膜中 4-(4氨基苯乙炔)苯之间因 $\pi-\pi$ 相互作用而形成相互聚集 ${ }^{[30]}$. 将上述制备的自组装单分子膜浸泡在一般有机溶剂(如 四氢呋喃、氯仿等)中, 其吸收强度变化很小, 说明 4-(4- 
氨基苯乙炔)苯基确以共价键接的方式组装在基片上, 得到稳定的单分子膜.

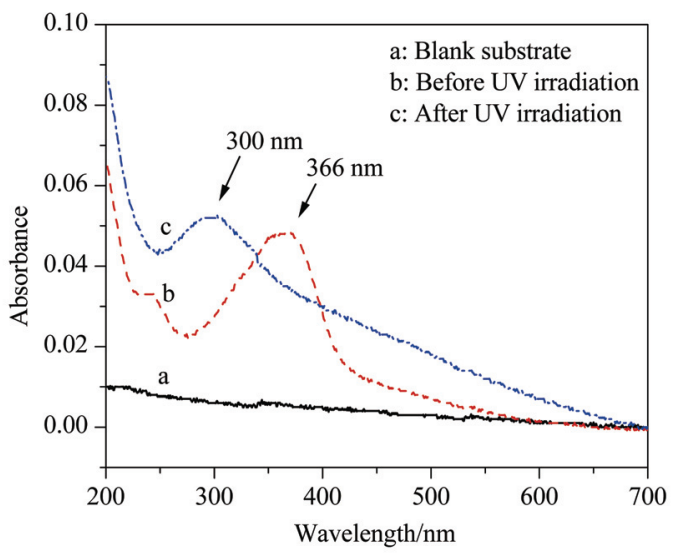

图 2 组装了 APEB- $\mathrm{N}_{2}^{+}$的单分子膜的紫外-可见吸收光谱

Figure 2 The UV-Vis absorption spectra of monolayers fabricated on a quartz with APEB- $\mathrm{N}_{2}^{+}$

\section{2 含有荧光探针萠功能超薄膜的制备及其发光性能}

4-(4-氨基苯乙炔)苯单分子膜的表面带有 $\mathrm{NH}_{2}$, 利 用 $\mathrm{NH}_{2}$ 的反应活性可进一步功能化制备功能超薄膜; 本
文采用两种方法将苂光探针萠引入单分子膜上，制备了 发光超薄膜(Figure 3). Figure 3 中 Precedure A 是通过 $\mathrm{NH}_{2}$ 和 $\mathrm{COOH}$ 间的酰胺化反应引入发色团萠. 与 1-萠正 丁酸溶液的 UV-Vis 曲线(曲线 a)相比, 功能超薄膜明显 出现发色团萠的特征吸收(曲线 $\mathrm{c}$ ), 说明已经成功修饰. Precedure B 采用重氮盐光化学反应方法修饰发色团萠: 先将单分子膜上 $\mathrm{NH}_{2}$ 重氮化 ${ }^{[29,30]}$ (Supporting Information, Figure S3), 其 UV-Vis 曲线在 $385 \mathrm{~nm}$ 处明显出现重 氮基 $\mathrm{N}_{2}^{+}$的特征吸收(曲线 $\mathrm{e}$ ) 然后 $\mathrm{N}_{2}^{+}$与萠丁酸的 $\mathrm{COO}^{-}$ 间因静电相互作用而使萠基团吸附在单分子膜上[曲线 $\mathrm{f}(1)$ ], 其 UV-Vis 吸收曲线中同时出现萠和重氮基 $\mathrm{N}_{2}^{+}$的 特征吸收; 紫外光辐照后, $385 \mathrm{~nm}$ 处重氮基的吸收峰消 失, 稠环萠的特征吸收峰依然存在 [曲线 $\mathrm{f}(2)]$, 说明单分 子膜上已修饰上发色团萠.

紫外光照后线形苯炔苯共价键接在基片上，共轭的 苯炔苯分子间距较小, 分子间会产生 $\pi-\pi$ 相互作用而发 生荧光猝灭. 因此苯炔苯共轭结构单分子膜并不发光 (Figure 3 曲线 1). 含有发色团萠的超薄膜明显具有发光 性能，出现了稠环化合物萠激基缔合物的苂光，发射波 长 $480 \mathrm{~nm}$ (Figure 3 曲线 2 和 3 ). 若仅通过柔性链将萠固 定在基片上的单分子膜只有萠单体的发射峰 ${ }^{[31]}$. 这是
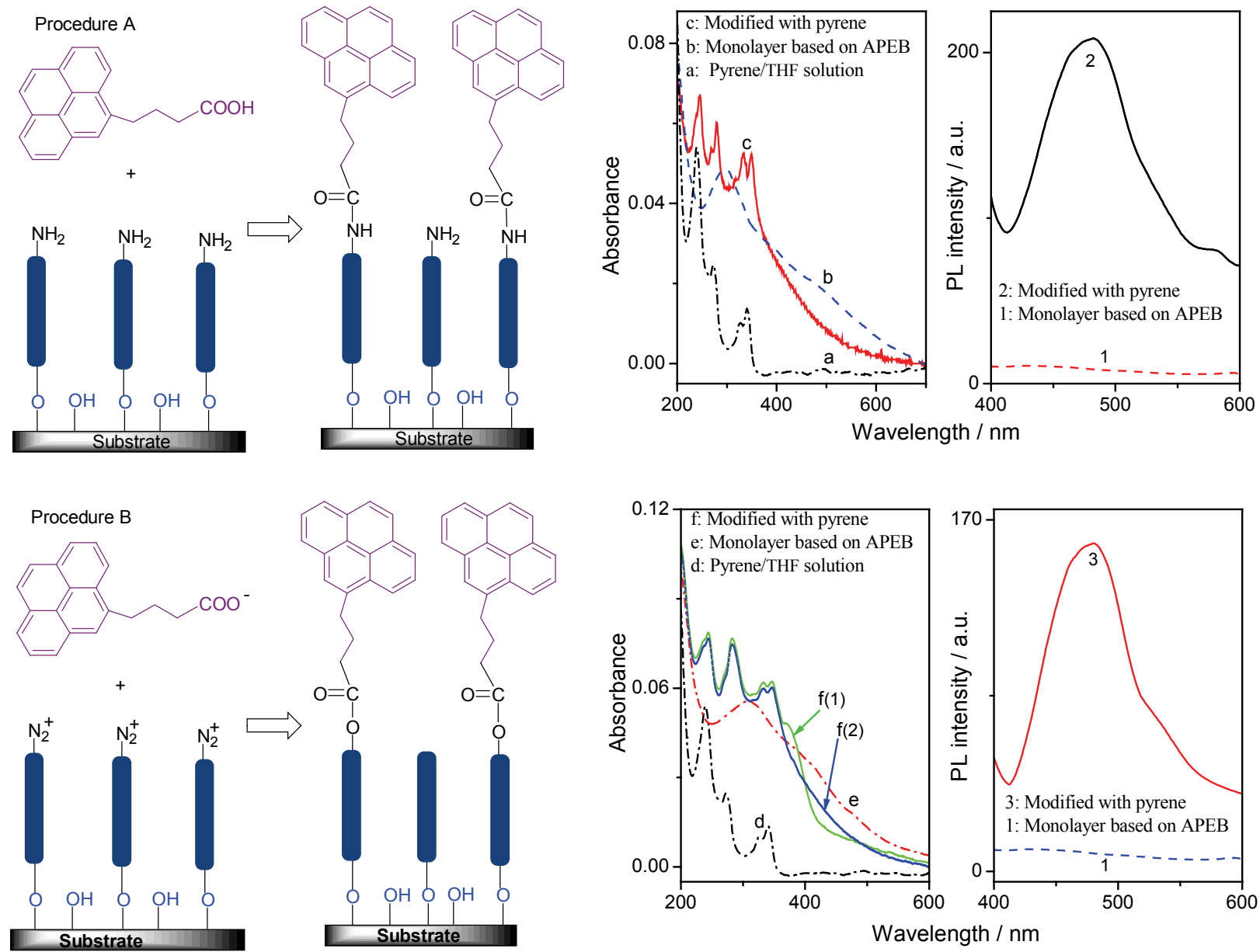

图 3 含有萠的功能超薄膜的制备及其紫外-可见吸收与菼光发射光谱 $\left(\lambda_{\mathrm{ex}}=340 \mathrm{~nm}\right)$

Figure 3 Preparation of functional ultra-thin films with pyrene and their UV-Vis absorption and fluorescence spectra $\left(\lambda_{\mathrm{ex}}=340 \mathrm{~nm}\right)$ 
因为将发色团萠修饰在苯炔苯单分子层上时, 因棒状苯 炔苯分子间 $\pi-\pi$ 相互作用使其分子之间排列规整 ${ }^{[30]}$, 在 进一步进行萠分子修饰时会起到诱导的作用, 使修饰到 苯炔苯单分子层上的萠分子倾向于更为规整的排列, 易 于形成面对面的空间构象 (g-g conformation)而形成基态 缔合物受激发射, 在 $480 \mathrm{~nm}$ 处出现苂光峰; 同时, 因位 阻作用和苯炔苯的刚性, 修饰在苯炔苯单分子膜上的芘 分子不会很密, 一定程度上也能避免稠环芳烃芘分子间 聚集诱导猝灭的发生.

两种不同修饰方式引入单分子膜上的荧光探针芘 的量略有差异, 根据两种方式修饰前后的特征紫外-可 见吸收峰相对强度比值的大小, 可知通过 $\mathrm{N}_{2}^{+}$与 $\mathrm{COO}^{-}$ 间静电相互作用修饰上的萠分子的量略大一些; 但该修 饰方式得到的功能薄膜的发光略弱一些, 主要源于更多 的稠环芳烃萠修饰到薄膜上后, 萠分子密度增大, 激发 态时也易发生分子间的电子能量转移而使荧光减弱.

\section{3 功能超薄膜对硝基苯胺的荧光响应}

染料工业极为重要的中间体, 硝基苯胺具有比较强 的毒性, 所以实现对硝基苯胺的高灵敏检测非常必要. 在已知各类检测方法中, 由于硝基芳烃易于与电荷给体 的苂光化合物、共轭聚合物、齐聚物间产生电荷转移络 合作用而改变其发光特性 ${ }^{[18,20]}$, 从而采用光谱法实现硝 基苯类化合物的检测一直受到关注. 本文选择硝基苯 胺(NA)的三种同分异构体: 邻-硝基苯胺、间一硝基苯 胺、对-硝基苯胺 $(o-\mathrm{NA}, m-\mathrm{NA}, p-\mathrm{NA})$ 作为猝灭剂, 考察 不同取代基位置时 NA 对功能薄膜发光性质的影响. 将 制备的功能超薄膜浸泡在 $\mathrm{CHCl}_{3}$ 以及 $o-\mathrm{NA}, m-\mathrm{NA}$, $p$-NA 的 $\mathrm{CHCl}_{3}$ 溶液 $\left(1 \times 10^{-4} \mathrm{~mol} \cdot \mathrm{dm}^{-3}\right)$ 中 $10 \mathrm{~min}$, 取出 后用 $\mathrm{CHCl}_{3}$ 清洗, 进行荧光测试. 结果表明, 在 $\mathrm{CHCl}_{3}$ 中浸泡后的超薄膜发光强度与浸泡前基本不变, 在 $\mathrm{NA} / \mathrm{CHCl}_{3}$ 溶液浸泡后, 虽然功能薄膜的发射波长不变, 但苂光强度却有明显下降, 这是因为电荷受体 NA 与富 电的稠环萠之间形成了电荷转移络合物使激发态的电 子能量发生转移, 功能超薄膜的非辐射能量损耗增加, 荧光被猝灭 ${ }^{[18,20]}$; 取代基相对位置不同的 NA 对功能薄 膜的荧光猝灭有明显差异, $p$-NA, $o$-NA, $m$-NA 的猝灭百 分率依次为 $54.8 \%, 39.3 \%, 6.1 \%$ (Figure $4 \mathrm{~A}$ ).

硝基苯胺中, 硝基和氨基都能与苯环构成大的 $\pi$-共 轭结构, 两者的相对取代位置不同会影响分子内共轭和 电子云偏移程度. 氨基是强给电基团, 其共轭效应使苯 环电子云密度增大，电子在邻对位富集，而氨基本身显 正电; 相反硝基是强吸电基团, 其诱导作用使苯环带正 电, 且与苯环共轭形成大 $\pi$ 键, 使苯环邻对位显正电, 而其本身显负电. 对于 $p$-NA, 两者的共轭作用和硝基 的诱导作用使得对位的氨基显正电最明显, 电子云发生 明显偏移, 带正电一端更易与富电的萠激发态接触发生 电荷转移猝灭, 明显降低功能薄膜的发光强度; $o$-NA 中 两基团共轭作用和硝基的诱导作用比 $p$-NA 小; $m$-NA 则
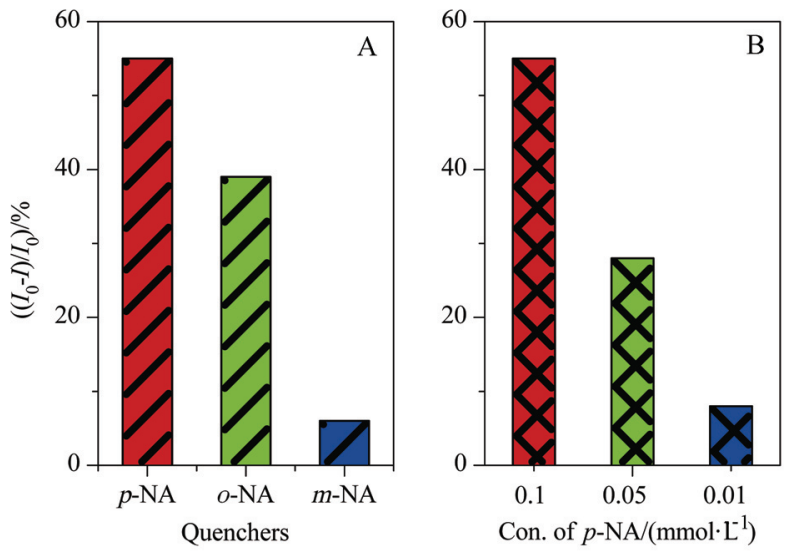

图 4 硝基苯胺对含萠功能超薄膜的苂光猝灭

Figure 4 The fluorescence quenching of NA to the functional ultra-thin film with pyrene

只有诱导作用, 所以 NA 的电子云偏移程度按 $p$-NA, $o$-NA, $m$-NA 依次减弱，与薄膜上电荷给体萠之间的电 荷转移效率亦是如此. $p$-NA 的浓度也明显影响对薄膜 的荧光猝灭程度, NA 浓度越低, 能够和电荷受体萠基 团之间形成的电荷转移络合物的量越小, 薄膜的苂光猝 灭程度也随之降低(Figure 4B).

为了考察其荧光响应的重复性, 将经过 $p$-NA 猝灭 后的功能薄膜用 $\mathrm{CHCl}_{3}$ 进行浸泡清洗, 清洗后薄膜的荧 光强度有所增加, 但不能回复到原有的发光强度, 主要 因为电子受体 $p$-NA 与富电的萠之间因 $\pi-\pi$ 相互作用会 生成稳定的电荷转移络合物, 即使用 $\mathrm{CHCl}_{3}$ 进行浸泡清 洗，只能除掉部分猝灭剂，所以超薄膜的苂光不能完全 恢复.

\section{3 结论}

利用重氮基的光分解活性、通过自组装的方法将共 轭的 4-(4-氨基苯乙炔)苯修饰在带有负电荷的石英基片 上制备了单分子膜; 利用单分子膜上 $\mathrm{NH}_{2}$ 的反应活性, 通过化学反应或静电自组装的方式, 用酰胺键或酯键将 发色团萠修饰在单分子膜上，构筑了含有苂光探针芘的 功能超薄膜; 含有苯炔苯的单分子膜因共轭结构之间的 相互作用易于发生非辐射能量的转移而不发光, 但两种 修饰方式得到的含苂光探针芘的功能超薄膜都具有较 好的发光性能, 最大发射波长为 $480 \mathrm{~nm}$; 并且, 该发光 超薄膜对不同取代位置的电荷受体硝基苯胺的荧光响 应具有明显差异，可以作为硝基苯胺同分异构体检测的 传感器材料.

\section{4 实验部分}

\subsection{4-(4-氨基苯乙炔基)苯重氮盐的制备}

4-(4-氨基苯乙炔基)苯重氮盐(APEB- $\mathrm{N}_{2}^{+}$)参考文献 的方法制备 ${ }^{[22,27]}$, 合成路线见 Scheme 1. 


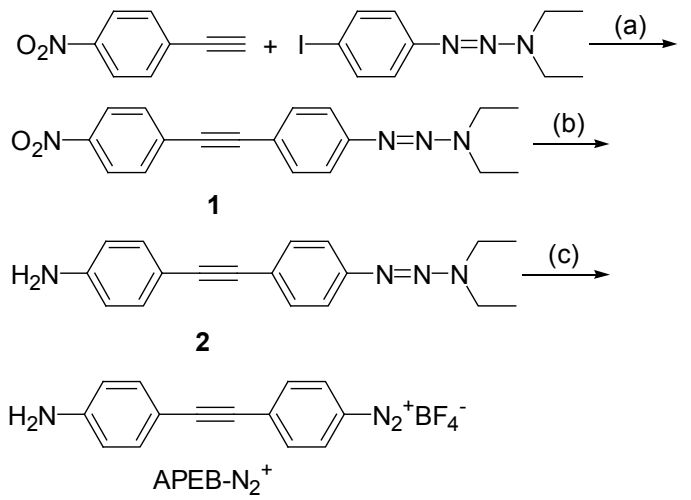

(a) $\mathrm{Pd}\left(\mathrm{PPh}_{3}\right)_{2} \mathrm{Cl}_{2}$, Cul/triethylamine; (b) $\mathrm{Zn}$, ammonia/acetone;

(c) $\mathrm{HBF}_{4}$ /acetonitrile

图式 1 4-(4-氨基苯乙炔基)苯重氮盐(APEB- $\mathrm{N}_{2}^{+}$)的合成 Scheme 1 Synthesis of APEB- $\mathrm{N}_{2}^{+}$

以对硝基苯乙炔和 1-(4-碘苯基)-3,3-二乙基三氮烯 为原料, 采用 Sonogashira 偶联反应合成 4-[4-(3,3-二乙 基三氮烯)-苯乙炔基]硝基苯(1)，产率 $61 \%$. ${ }^{1} \mathrm{H} \mathrm{NMR}$ $\left(\mathrm{CDCl}_{3}, 400 \mathrm{MHz}\right) \delta: 1.27(\mathrm{t}, J=6.8 \mathrm{~Hz}, 6 \mathrm{H}), 3.80$ (q, $J=$ $7.2 \mathrm{~Hz}, 4 \mathrm{H}), 7.44(\mathrm{~d}, J=8.6 \mathrm{~Hz}, 2 \mathrm{H}), 7.53$ (d, $J=8.5 \mathrm{~Hz}$, 2H), 7.65 (d, $J=8.8 \mathrm{~Hz}, 2 \mathrm{H}), 8.22$ (d, $J=8.8 \mathrm{~Hz}, 2 \mathrm{H})$; IR (KBr) $v: 3040,2970,2240,1592,1500,1380 \mathrm{~cm}^{-1}$.

以 $\mathrm{Zn}$ /氨水为还原剂, 4-[4-(3,3-二乙基三氮烯)-苯乙 炔基]硝基苯被还原生成 4-[4-(3,3-二乙基三氮烯)-苯乙 炔基]氨基苯(2), 产率 70\%. ${ }^{1} \mathrm{H} \mathrm{NMR}\left(\mathrm{CDCl}_{3}, 400 \mathrm{MHz}\right)$ $\delta$ : 1.25 (t, $J=6.84 \mathrm{~Hz}, 6 \mathrm{H}), 3.80$ (q, $J=7.2 \mathrm{~Hz}, 4 \mathrm{H}), 6.63$ (d, $J=8.4 \mathrm{~Hz}, 2 \mathrm{H}), 7.34$ (d, $J=8.5 \mathrm{~Hz}, 2 \mathrm{H}), 7.38(\mathrm{~d}, J=8.5$ $\mathrm{Hz}, 2 \mathrm{H}), 7.46$ (d, $J=8.5 \mathrm{~Hz}, 2 \mathrm{H})$; IR (KBr) v: 3450, 2980, $2240,1603,1500 \mathrm{~cm}^{-1}$.

将 4-[4-(3,3-二乙基三氮烯)-苯乙炔基]氨基苯加入 $2 \%$ 的氟嗍酸乙腈溶液中室温下水解, 即可得到 4-(4-氨 基苯乙炔)苯基重氮盐(APEB- $\mathrm{N}_{2}^{+}$)(Supporting Information, Figure S1, S2).

\section{2 功能薄膜的制备}

\subsection{1 单分子膜的制备}

将预处理的表面带有负电荷的石英基片 ${ }^{[18,29,32]}$ 在 APEB- $\mathrm{N}_{2}^{+}$/乙腈溶液中避光浸泡 $30 \mathrm{~min}$, 取出后在纯净 的乙腈中浸泡清洗 $2 \mathrm{~min}, \mathrm{~N}_{2}$ 吹干后置于紫外光 $(250 \mathrm{~W}$, $245 \mathrm{~nm}$ )下辐照 $10 \mathrm{~min}$, 冷却至室温, 浸入纯净的三氯甲 烷中超声清洗 $2 \mathrm{~min}$, 再用 $\mathrm{N}_{2}$ 吹干, 完成单分子层制备 (Figure 1a).

\section{2 .2 单分子膜上 $\mathrm{NH}_{2}$ 的重氮化}

将修饰了 4-(4-氨基苯乙炔)苯基单分子层的石英片 放入 $8 \mathrm{~mol} \cdot \mathrm{dm}^{-3}$ 的盐酸溶液中, 冰浴下反应 $10 \mathrm{~min}$, 然 后加入 $\mathrm{NaNO}_{2}$ 水溶液 $\left(0.7 \mathrm{~mol} \cdot \mathrm{dm}^{-3}\right)$, 避光反应 $30 \mathrm{~min}$, 取出石英片, 用去离子水洗涤, $\mathrm{N}_{2}$ 吹干后避光保存备用, 重氮化后单分子膜在 $385 \mathrm{~nm}$ 出现了重氮基的特征 UV-Vis 吸收峰(Supporting Information, Figure S3).

\subsection{3 带有荧光团萠的功能超薄膜的制备}

(1)将共价键接了 4-(4-氨基苯乙炔)苯基的石英片浸 入 1-萠正丁酸的 $\mathrm{THF}$ 溶液 $\left(0.025 \mathrm{~mol} \bullet \mathrm{dm}^{-3}\right)$ 中, 加入 DCC, TsOH, DMAP，室温反应 $12 \mathrm{~h}$ ，将石英片取出，用 二氯甲烷和 THF 浸泡清洗, $\mathrm{N}_{2}$ 吹干得到通过酰胺化反应 制备的功能超薄膜(Figure 3: Procedure A).

(2)将组装有 4-(苯乙炔基)苯基重氮盐的石英片避光 浸入 1 -萠正丁酸钠水溶液 $\left(1 \times 10^{-3} \mathrm{~mol} \cdot \mathrm{dm}^{-3}\right)$ 中浸泡 30 $\min$, 取出石英片, 用紫外光照射, 然后依次用去离子 水、三氯甲烷超声清洗, $\mathrm{N}_{2}$ 吹干后得到组装方法制备的 功能薄膜(Figure 3: Procedure B).

\section{References}

[1] Sagiv, J. J. Am. Chem. Soc. 1980, 102, 92.

[2] Ulman, A. Chem. Rev. 1996, 96, 1533.

[3] Ulman, A. Acc. Chem. Res. 2001, 34, 855.

[4] Sullivan, T. P.; Huck, W. T. S. Eur. J. Org. Chem. 2003, (1), 17.

[5] Brandow, S. L.; Chen, M. S.; Dulcey, C. S.; Dressick, W. J. Langmuir 2008, 24, 3888

[6] Choi, S. H.; Kim, B.; Frisbie, C. D. Science 2008, 320, 1482.

[7] McArthur, E. A.; Ye, T.; Cross, J. P.; Petoud, S.; Borguet, E. J. Am. Chem. Soc. 2004, 126, 2260.

[8] Xu, Q. M.; Ma, H.; Yip, H.; Jen, A. K.-Y. Nanotechnology 2008, 19, 135605.

[9] Valkenier, H.; Huisman, E. H.; Hal, P. A.; Leeuw, D. M.; Chiechi, R. C.; Hummelen, J. C. J. Am. Chem. Soc. 2011, 133, 4930

[10] Azam, M. S.; Fenwick, S. L.; Gibbs, D. J. M. Langmuir 2011, 27, 741.

[11] Fuentes, O. A.; Ferri, T.; Frasconi, M.; Paolini, V.; Santucci, R. Angew. Chem. Int. Ed. 2011, 50, 3457.

[12] Gao, Y.; Xu, G. H.; An, Y. Acta Phys.-Chim. Sin. 2010, 26, 2211. (高源, 徐国华, 安越, 物理化学学报, 2010, 26, 2211.)

[13] Figueira-Duarte, T. M.; Simon, S. C.; Wagner, M.; Druzhinin, S. I.; Zachariasse, K. A.; Müllen, K. Angew. Chem., Int. Ed. 2008, 47, 10175.

[14] Hu, J. Y.; Era, M.; Elsegood, M. R. J.; Yamato, T. Eur. J. Org. Chem. 2010, 72.

[15] Wu, K. C.; Ku, P. J.; Lin, C. S.; Shih, H. T.; Wu, F. I.; Huang, M. J.; Lin, J. J.; Chen, I. C.; Cheng, C. H. Adv. Funct. Mater. 2008, 18, 67.

[16] Chen, Y.; Wang, Y. Y.; Yu, P.; Liu, Z. Q.; Fang, Q. Chin. J. Org. Chem. 2012, 32, 589. (陈芗, 王园园, 于萍, 刘志强, 方奇, 有机 化学, 2012, 32, 589).

[17] Zhang, S. J.; Lu, F. T.; Gao, L. N.; Ding, L. P.; Fang, Y. Langmuir 2007, 23, 1584.

[18] Liu, T. H.; Ding, L. P.; Zhao, K. R.; Wang, W. L.; Fang, Y. J. Mater. Chem. 2012, 22, 1069.

[19] Kepley, L. J.; Crooks, R. M.; Ricco, A. J. Anal. Chem. 1992, 64 3191

[20] Li, H. H.; Kang, J. P.; Ding, L. P.; Lu, F. T.; Fang, Y. J. Photochem. Photobiol. Chem. 2008, 197, 226.

[21] Ding, L. P.; Cuil, X. A.; Han, Y. N.; Lu, F. T.; Fang, Y. J. Photochem. Photobiol. Chem. 2007, 186, 143.

[22] Liu, T. H.; Nie, Y. X.; He, G.; Zhang, Y.; Ding, L. P.; Fang, Y. Chem. J. Chin. Univ. 2010, 31, 524. (刘太宏, 聂云霞, 何刚, 张媛, 丁立平, 房喻, 高等学校化学学报, 2010, 31, 524.)

[23] He, G.; Yan, N.; Kong, H. Y.; Yin, S. W.; Ding, L. P.; Qu, S. X.; Fang, Y. Macromolecules 2011, 44, 703.

[24] Acharya, J. R.; Zhang, H. T.; Li, X.; Nesterov, E. E. J. Am. Chem. Soc. 2009, 131, 880 .

[25] Hsiao, H. Y.; Chen, M. L.; Wu, H. T.; Huang, L. D.; Chien, W. T.; Yu, C. C.; Jan, F. D.; Sahabuddin, S. K.; Chang, T. C.; Lin, C. C. Chem. Commun. 2011, 47, 1187.

[26] Li, Y.; Wang, J.; Cai, C. Z. Langmuir 2011, 27, 2437.

[27] Flatt, A. K.; Chen, B.; Tour, J. M. J. Am. Chem. Soc. 2005, 127, 8918.

[28] Zhi, J. G.; Tian, X. L.; Zhao, W.; Shen, J. B.; Tong, B.; Dong, Y. P. J. Colloid Interface Sci. 2008, 319, 270.

[29] Zhao, W.; Tong, B.; Pan, Y. X.; Shen, J. B.; Zhi, J. G.; Shi, J. B.; 
Dong, Y. P. Langmuir 2009, 25, 11796

[30] Zhao, W.; Tong, B.; Zhi, J. G.; Pan, Y. X.; Shen, J. B.; Shi, J. B.; Dong, Y. P. Acta Phys.-Chim. Sin. 2010, 26, 822. (赵玮, 佟斌, 支 俊格, 潘月秀, 申进波, 石建兵, 董宇平, 物理化学学报, 2010, $26,822)$.

[31] Li, H. H.; Lü, F. T.; Zhang, S. J.; He, G.; Fang, Y. Chin. Sci. Bull.
2008, 53, 394. (李慧慧，吕风婷，张淑娟，何刚，房喻，科学通报， 2008, 53, 394).

[32] Pan, Y. X.; Tong, B.; Zhi, J. G.; Zhao, W.; Shen, J. B.; Shi, J. B.; Dong, Y. P. Acta Chim. Sinica 2009, 67, 2779. (潘月秀, 佟斌, 支 俊格, 赵玮, 申进波, 石建兵, 董宇平, 化学学报, 2009, 67, 2779).

(Cheng, B.; Lu, Z.) 